\title{
Formación matemática sistematizada a partir del enfoque ciencia, tecnología y sociedad, en el perfil ingenieril
}

Faustino, Arnaldo; Pérez Sánchez, Néreyda; Diéguez Batista, Raquel

Formación matemática sistematizada a partir del enfoque ciencia, tecnología y sociedad, en el perfil ingenieril

Revista Educación, vol. 43, núm. 1, 2019

Universidad de Costa Rica, Costa Rica

Disponible en: http://www.redalyc.org/articulo.oa?id=44057415006

DOI: https://doi.org/10.15517/revedu.v43i1.28233

Esta obra está bajo una Licencia Creative Commons Atribución-NoComercial-SinDerivar 3.0 Internacional. 


\section{Formación matemática sistematizada a partir del enfoque ciencia, tecnología y} sociedad, en el perfil ingenieril

Systematic mathematical approach formation from science, technology and society at the engineering profile

Arnaldo Faustino 1

Universidad Óscar Ribas (UÓR), Angola

arnaldo.faustino19@gmail.com

http://orcid.org/0000-0003-3197-5348

\section{Néreyda Pérez Sánchez 2}

Universidad Máximo Gómez Báez (UNICA), Ciego de

Ávila, Cuba

nereyda@rect.unica.cu

\section{Raquel Diéguez Batista 3}

Universidad Máximo Gómez Báez (UNICA), Ciego de

Ávila, Cuba

raqueldb1961@yahoo.es
DOI: https://doi.org/10.15517/revedu.v43i1.28233

Redalyc: http://www.redalyc.org/articulo.oa?id=44057415006
Recepción: 15 Marzo 2017

Aprobación: 24 Noviembre 2018

\section{ReSUMEN:}

La actual investigación se desarrolló como parte de una formación doctoral y su ejecución en el desempeño pedagógico desde 2013 hasta 2018, la cual se socializa a través de un artículo científico, a partir del proceso de formación matemática. Se posibilita metodológicamente el perfeccionamiento del proceso formativo, a fin de elevar los niveles de sistematización cognitiva, sustentado en una relación establecida entre la abstracción matemática científica y la generalización matemática investigativa; como posición epistemológica para solución de problemas profesionales, enfocados en la Ciencia, Tecnología y Sociedad (CTS). Su implementación parcial se realizó en los estudiantes del tercer y cuarto año académico, en las carreras de Ingeniería Civil, Informática, Electrónica, Telecomunicación e Información en las Instituciones de la Enseñanza Superior (IES) angoleñas. Además, los resultados alcanzados en la pesquisa con la utilización del método histórico-lógico, el análisis documental y la técnica de muestreo aleatorio estatificado son valorados por algunos profesores, quienes tuvieron relación directa con la formación matemática, quienes posibilitaron el procesamiento de la información, a través del empleó del software IBM SPSS Statistics 20; logrando una transformación cuali-cuantitativa en el proceso de enseñanza-aprendizaje de la Matemática, mediante proyecciones investigativas de mayor pertinencia, teniendo en cuenta las demandas sociales y la adecuada implementación de los aportes científicos reconocidos por la Comunidad Científica. Así se facilitó la inserción en el contexto, en función de resolver las diversas problemáticas de la profesión y del territorio donde se reside, provocando cambios en el rol estudiantil, la economía, y en la prestación de los servicios.

Palabras ClaVE: Formación matemática, ciencia, tecnología, sociedad (CTS), Angola.

\section{Abstract:}

The current research was developed as part of a doctoral training and its execution in pedagogical performance from 2013 to 2018. It is socialized through a scientific article, based on a mathematical training process. The methodological perfection of

\section{NOTAS DE AUTOR}

1 Vice Rector para Asuntos Científicos y de Extensión Universitaria de la Universidad Óscar Ribas (UÓR), Luanda Angola. Doctor en Ciencias Pedagógicas, Universidad de Oriente, (UO) y Universidad Máximo Gómez Báez (UNICA), Cuba. Identificador de autor en Redalyc: https:// www.redalyc.org/autor.oa?id=18523.

2 Vice Rector para Asuntos Científicos y de Extensión Universitaria de la Universidad Máximo Gómez Báez (UNICA), Ciego de Ávila, Cuba.

3 Profesora titular de Matemática de la Facultad de Ingeniería, de la Universidad Máximo Gómez Baéz (UNICA), Ciego de Ávila, Cuba. 
the formative process is possible, in order to raise the levels of cognitive systematization, based on a relationship established between scientific mathematical abstraction and investigative mathematical generalization; as an epistemological position to solve professional problems, focused on Science, Technology and Society (CTS). Its partial implementation was carried out on the students of the third and fourth academic year, in the careers of Civil Engineering, Information Technology, Electronics, Telecommunications and Information in the Institutions of Higher Education (IES) in Angola. In addition, the results achieved in the research with the use of the historical-logical method, the documentary analysis and the static randomized sampling technique are valued by some professors, who had a direct relationship with the mathematical training, and made possible to process the information, through the use of IBM SPSS Statistics 20 software. These results achieved a qualitative-quantitative transformation in the teaching-learning process of Mathematics, through research projections of greater relevance, taking into account social demands and the adequate implementation of scientific contributions recognized by the Scientific Community. This facilitated the insertion in the context, in order to solve the various problems of the profession and the territory where it resides, causing changes in the students' role, the economy, and the service provision.

KEYWORDS: Mathematics education, science, technology, society (STS), Angola.

\section{INTRODUCCIÓN}

La evolución científica-tecnológica, imponen retos a las formaciones matemáticas de formar profesionales responsables y comprometidos, con toda construcción científico-cognitiva para transformar el contexto sociocultural. Donde los avances tecnológicos han revolucionado los procesos productivos aceleradamente, proporcionando diversas ofertas con nuevas estructuras epistémicas. Sin embargo, su utilización pertinente requiere una apreciación holística, la cual permita obtener alternativas viables para solucionar problemáticas cotidianas. Por ello es necesario perfeccionar componentes académicos-investigativos en los sistemas educacionales superiores.

Sucede entonces, que las Instituciones de Educación Superior (IES), públicas, público-privadas y privadas en la sociedad angoleña para perfeccionarse en medio de la globalización, demandan como misión; la formación matemática de profesionales implicados en el desarrollo de los avances investigativos del mundo contemporáneo; donde el eje Ciencia, Tecnología y Sociedad (CTS), las políticas del desarrollo del Consejo Nacional de la Ciencia, Tecnología e Innovación (CNCTI), la Dirección Nacional de Desarrollo Tecnológico e Innovación (DNDTI); el Centro Tecnológico Nacional (CTN) en la sociedad angoleña contribuye al desempeño exitoso de intelectuales y empresarios. Todo lo cual implica que las Universidades públicas, públicas-privadas y privadas como gestoras de culturas, potencien "la constante elevación del nivel científico de sus profesionales "como una tarea determinada, específicamente al fortalecimiento de la formación científica (Fuentes, 2009 p. 476).

Así el enfoque científico de ciencia, tecnología y sociedad adquiere hoy una notable importancia, porque las políticas para dinamizar el Paquete de Ciencia y Tecnología (PCT) para el siglo XXI en Angola, posibilita desde los conocimientos científicos-tecnológicos, relevantes perspectivas sociales generadoras del desarrollo, lo cual debe ser reconocido pedagógicamente e implementarse didácticamente en los procesos formativos; estimulando independencias cognitivas, creatividad e implicaciones socioculturales. Donde las apreciaciones teóricas-prácticas pueden ser corroboradas con mayores índices de validación, van logrando modelaciones a cortos plazos que facilitan los patrones indagativos-innovativos pertinentemente para estimular el desarrollo de las políticas científico-tecnológicas bien como las educativas, las cuales deben desplazar los viejos abordajes conceptuales con los cuales operaban en el pasado y sustituirlo por ideas contemporáneas para una inevitable comprensión de los procesos de innovación social. Por esta razón, "hay que trabajar para la innovación", pero colocando por delante los objetivos del Ministerio de la Enseñanza Superior Ciencia Tecnología e Innovación (MESCTI) en la sociedad angoleña que ella debe atender (Núñez, 1999. p 244).

Sin embargo, los resultados del diagnóstico fáctico realizado entre 2013 y 2018 a los estudiantes de las carreras de perfil ingenieril, desde la Ingeniería Civil, Informática, Electrónica, Telecomunicación e Información; muestran la insuficiente utilización de los métodos de la ciencia y técnica, así como también 
el desarrollo de los conocimientos a partir de la solución de problemas reales de la sociedad, lo cual requiere de una adecuada formación matemática sistematizada, como soporte para resolver las problemáticas profesionales que limitan no solo la orientación de los estudiantes en la realización de las tareas investigativas, sino la aplicación de los conocimientos adquiridos, a situaciones de la realidad en que se desempeñan.

Este análisis previo posibilitó, además, apreciar las manifestaciones de una deficiente participación activa de los estudiantes, en su vínculo con las nuevas problemáticas de la producción de los servicios públicos, público-privados y privados. Así que, resulta oportuno plantear como objetivo general de la presente investigación, elevar los niveles de sistematización cognitiva de los estudiantes, mediante el desarrollo de una proyección metodológica, dinamizada por el enfoque CTS, para contribuir a minimizar las limitaciones presentadas en los procesos indagativos.

\section{REFERENTES CONCEPTUALES}

\subsection{Retos de la formación matemática en las carreras de perfil ingenieril a partir del enfoque de Ciencia, Tecnología y Sociedad}

Las carreras de perfil ingenieril, donde se encuentran Civil, Informática, Electrónica, Telecomunicación e Información, deben preparar profesionales capaces de realizar, diagnósticos, asesorías, investigaciones e implementaciones de resultados investigativos en las esferas sociales de la producción de los servicios públicos, público-privados y privados, para resolver las problemáticas existentes de forma pertinente y eficaz. Estas acciones tal como refiere Faustino, Pérez Diéguez (2012), citados por Faustino (2014), requieren del conocimiento de los métodos de la investigación empleados en el desarrollo de la ciencia como el análisis-síntesis, deducción-inducción y abstracción-concreción en la solución de los problemas. Además de la analogía-modelación, axiomatización y demostración que se utilizan en la formación matemática los cuales se enriquecen con tecnologías para el procesamiento de la información siendo estos, soportes usados por los estudiantes para la toma adecuada de las decisiones oportunas en el análisis, presentación e interpretación de los problemas profesionales en las empresas públicas públicas-privadas y privadas.

Los estudiantes de las carreras de perfil ingenieril, necesitan también de un fortalecimiento cognitivo en su formación matemática a partir de la sistematización, en estrecho vínculo con el enfoque CTS. Por ello, la formación matemática sistematizada en el presente estudio, es el proceso orientado a desarrollar en el estudiante, una visión científica de los requerimientos de su contexto que incluye, fuera del sistema de conocimientos matemáticos, los juicios, razonamientos, opiniones, criterios y convicciones. Mientras el enfoque CTS, que se asume en la actual investigación según Núñez (1999), citado por Fuentes (2009) determina el carácter crítico e interdisciplinar donde se estudia la dimensión de la ciencia y tecnología, tanto en lo que concierne a sus antecedentes como las consecuencias sociales, ambientales.

La adecuada aplicación del enfoque Ciencia, Tecnología y Sociedad en la formación matemática, constituye un problema; porque las políticas desarrolladas por el MESCTI, reconocen los aportes científicos y no implementan estrategias para los avances tecnológicos en la solución de los problemas sociales, los cuales limitan cognitivamente el desarrollo de diagnósticos, modelaciones, así como también la aplicación de alternativas que transformen contextos sociales desde dinámicas científicas.

En la presente investigación se concibe entonces, el eje Ciencia, Tecnología y Sociedad, en las carreras de perfil ingenieril como un proceso el cual permite la contextualización de una forma armónica, donde se aplican métodos de la ciencia y técnicas investigativas orientadas a lograr en los estudiantes una formación matemática, que les posibilite resolver problemáticas reales planteadas en su campo de acción profesional, a través de una sistematización de los contenidos matemáticos, a un nivel de generalidad, que le facilite aplicar los aportes de la ciencia y tecnología en su comunidad universitaria la cual es una representación humana 
y espacial que se establece entre los profesores, estudiantes, trabajadores de los servicios públicos, públicoprivados y privados, dentro de las instalaciones educativas; donde se desarrolla la docencia, la investigación, la práctica, así como también las actividades extensionistas en un tiempo determinado a partir de relaciones interpersonales.

No obstante, otros autores como García, Antonio (2001); Gómez; Mateos (2002); Acevedo; Vázquez; y Manassero (2003); Beckett; Margutti do Amaral (2005); Morell (2007); Núñez; Ortega; Mauro; Passailaigue; Fernando; Bayardo; Mauricio (2014), aprecian en el enfoque CTS de forma significativa, el rendimiento académico de los estudiantes y las características del proceso formativo. Así pues, la formación matemática debe promover diferentes aspectos de comprensión, sobre la relación de los temas matemáticos y el desarrollo tecnológico; además de contribuir a revertir los problemas de la preparación de los profesionales, adquiriendo en este sentido un mayor compromiso con los procesos de innovación en los sectores productivos de los servicios públicos, públicos-privados y privados en Angola.

Así, la formación matemática enfocada en los estudios CTS, pueden contribuir al desarrollo de una preparación pertinente y contextualizada, donde se posibilitan oportunidades y retos sociocognitivos. Sin embargo, esta perspectiva no se ha tenido en cuenta en los planes de estudios de la República de Angola para las carreras de perfil ingenieril. Se requiere, desarrollar en los estudiantes la integración de los contenidos matemáticos desde el enfoque CTS, de forma creadora, para fortalecer la argumentación de situaciones problémicas relacionadas con el objeto de trabajo y de la profesión como expresión del desarrollo de las capacidades profesionales en la solución de los problemas. Estos problemas son denominados como profesionales de acuerdo con la concepción expuesta por (Fuentes, 2009).

Donde se delimitan desde las dificultades, insuficiencias, debilidades, los espacios precisos a transformar, en un proceso de producción o de prestación de servicios públicos, público-privados y privados, presentes en una institución, empresa o centro laboral del perfil ingenieril, necesitado de acciones innovadoras, adaptativas a las condiciones reales del proceso productivo. En el cual, los estudiantes deben aplicar los conocimientos adquiridos de forma eficiente. Permitiéndole el desarrollo de habilidades laborales, a partir de las habilidades intelectuales del aprendizaje significativo que posibilita a la vez, la materialización de la teoría y su resignificación, mediante la argumentación científica de los problemas profesionales.

En la presente investigación se valora, la argumentación científica como un proceso integrado por la abstracción matemática científica y generalización matemática investigativa; donde se aporta un conjunto de razones que justifican, a modo de constatación, la veracidad de las cualidades y comportamientos reconocidos en los problemas profesionales. A través del uso de las tecnologías, donde se encuentran los asistentes matemáticos como: Mathcad professional, Wolfram Mathematica, Derive y los modelos estadísticos que facilitan la modelación de los problemas matemáticos para contribuir a solucionar las necesidades sociales.

Una razón más para pensar en la necesidad de usar los asistentes matemáticos en la solución de los problemas profesionales, es lo evidenciado en la Educación Superior angoleña, donde aún existen insuficiencias en el desempeño de los estudiantes de las carreras de perfil ingenieril. Para la solución de los problemas que se presentan en el marco de su labor social. Y al mismo tiempo precisan ser abordados desde el enfoque de Ciencia, Tecnología y Sociedad, en función de resolver lo demandado por los territorios.

Se debe lograr una adecuada valoración de los problemas profesionales y sociales desde el enfoque de Ciencia, Tecnología y Sociedad, donde la formación matemática no esté caracterizada por la fragmentación en la selección de métodos y técnicas para solución de problemas profesionales, como lo aprecia Guzmán (2008), en sus estudios. Por lo que se procede a resolver problemáticas de forma empírica y no desde la aplicación de los métodos de la ciencia y la técnica, limitando el enfoque totalizador a partir del cual, se desarrolla la modelación de los problemas matemáticos orientados al desarrollo del país.

La modelación de los problemas matemáticos requiere, de la obtención de información relacionada con el objeto de la profesión, mediante la aplicación de la argumentación científica. Siendo esta una categoría relevante para la transformación de procesos reales, manifestados en cualquier contexto, la cual, es apreciada 
significativamente por otros investigadores como Fuentes, Matos y Cruz (2004) quienes la valoran desde otras ciencias, para interpretar diversas situaciones y poder proyectar alternativas de solución con mayor precisión en su contexto cultural. En este sentido, es muy importante tomar en consideración lo históricocultural desarrollado por "Lev Semyonovich Vygotsky" ya citados por Faustino (2014), aunque no se expresa de manera explícita su contribución para dinamizar el desarrollo de la formación matemática sistematizada a partir del enfoque Ciencia, Tecnología y Sociedad, en el perfil ingenieril, se aprecia que algunos de sus principales presupuestos teóricos, constituyen sustentos indispensables para el desarrollo de este tipo de formación. Pues la enseñanza-aprendizaje de la Matemática enfocada en el eje Ciencia, Tecnología y Sociedad es un proceso a través del cual una misma información se relaciona, de manera no arbitraria y sustantiva, con un aspecto relevante en la estructura cognitiva del individuo para desarrollar el pensamiento investigativo basada en un constructivismo social.

Sin embargo, es imprescindible revelar también cómo en la modelación de los problemas matemáticos, la interpretación enfocada en el contexto social y en los estudios CTS, es una acción dinamizadora que, permite en la práctica la representación ideal del objeto modelado, las estructuras externas de sus procesos, relaciones dialécticas, los aspectos medulares de sus limitaciones, las fortalezas que prevalecen y los espacios o rasgos típicos las cuales facilitan su transformación. Sin ella, es imposible determinar las causales y resultantes de una problemática profesional y social. Así mismo, la identificación de componentes renovadores de etapas de carácter sistematizador o generalizador, para la transformación del objeto investigado; a partir de una coherente argumentación científica la cual justifique su potencialidad y pertinencia.

Por ello se asume entonces, en la presente investigación a la modelación en la formación matemática, como una habilidad intelectual necesaria para el ejercicio de la profesión que facilita la representación ideal de una situación, mediante su interpretación. Se vislumbran las vías a seguir y el propósito a alcanzar para la solución a las problemáticas de la profesión y la sociedad, sobre la base de la delimitación de sus aspectos identificativos. En el caso del perfil ingenieril en la sociedad angoleña es insustituible, por la utilidad que proporciona en el desarrollo de las valoraciones, aproximaciones y proyecciones técnicas; donde el análisis requiere de la comprensión que facilita los procesos interpretativos de la realidad para una sociología funcionalista de las Ciencias.

Así, en las carreras de perfil ingenieril, hay un reconocimiento de la necesidad de formar profesionales cuya cultura esté de acuerdo a la realidad científica-tecnológica en la cual se desarrolla. Esto también se puede apreciar, en las reformas educativas que se está dando en las instituciones educativas angoleñas, como la introducción de las carreras de Ingeniería Informática y la promoción del conocimiento computacional en la formación matemática para que el estudiante tenga una preparación en el futuro ejercicio de su profesión. Por ello, aunque no se expresa de manera explícita en los supuestos teóricos sobre la integración de nuevas prácticas educativas en la profesión, del pedagogo "Johann Pestalozzi“ en la formación matemática enfocada en el eje Ciencia, Tecnología y Sociedad, en la sociedad angoleña se asume en la presente investigación que se necesita encontrar en la formación matemática, formas adecuadas que promuevan el desarrollo de los niveles de sistematización cognitiva de los estudiantes conducentes a una sociología del conocimiento científico.

Dicho en otros términos, si realmente se aspira a que los estudiantes, en la sociedad angoleña, tengan una preparación adecuada en el futuro ejercicio de su profesión; es necesario sistematizar los conocimientos sobre la Ciencia, Tecnología y Sociedad. Teniendo en cuenta los impactos sociales, a fin de apoyar el desarrollo económico y la estabilidad del país, lo cual demandan la existencia de una preparación profesional y técnicas, en las Instituciones de Educación Superior en el perfil ingenieril. Suponiendo la identificación y tratamiento metodológico a los conocimientos matemáticos. Cuestión que se debe poner en práctica no solo para estos conocimientos, sino que pueden ser aplicados en áreas sociohumanísticas; donde se estudian procesos eminentemente sociales, pero requieren de una apreciación sustentada en la epistemología ${ }^{[1]}$ de la ciencia y técnica. 
En ello, como lo argumenta el eminente pedagogo Paulo Freire, ya citado por Chaves (2004), en sus escritos los estudiantes necesitan estar conscientes de su educación para desarrollar sus investigaciones partiendo de las potencialidades propias del contenido, a fin de propiciar la formación y desarrollo de las capacidades profesionales para resolver problemáticas reales. Donde realicen valoraciones reales de las problemáticas de la profesión, a partir del reconocimiento de las características sociales que la delimitan de los recursos científicostécnicos necesarios para su transformación. De esta forma se permite asumir una mayor autonomía en la toma de decisiones adecuadas desde el eje Ciencia Tecnología y Sociedad de forma holística.

En particular, en las carreras de Ingeniería Civil, Informática, Electrónica, Telecomunicación e Información, los problemas que se presentan en el marco de su labor social tienen la particularidad de ser abordados desde la perspectiva cuali-cuantitativa como un todo, lo cual las diferencia de otras carreras donde se manifiesta solamente una de estas. Por tanto, se necesita enfocar el eje Ciencia Tecnología y Sociedad en la formación matemática y "enseñar todo a todos" como se plantea en la Didáctica Magna de Jean Amos Komenský, (Comênio, 1966). Lo cual permitirá lograr una perfecta combinación en el análisis de datos cuali-cuantitativos en la formación matemática que están relacionados con el carácter de las Ciencia en los problemas sociales.

En la presente investigación, la formación matemática sistematizada enfocada en el eje Ciencia Tecnología y Sociedad, busca la información partiendo de una suposición predictiva acerca de los resultados esperados para obtener como solución del problema planteado, sobre la base de una primera observación de los hallazgos que se muestran superficialmente en la realidad contextual. Esta suposición orienta el sentido de la Ciencia, Tecnología y Sociedad a nuevas informaciones cuyo resultado confirma o refuta la predicción anterior, a través de argumentos que sirven tanto para orientar al sujeto en su pesquisa o como para proveerlo de juicios argumentativos.

Entonces estos, adquieren una cultura científica mediante la formación matemática. Ellos son reconocidos por los autores como Cuicas, Debel, Casadei, Álvarez (2007); Wongo; Faustino; Pérez (2013); Faustino; Pérez; Diéguez (2014), quienes aprecian la cultura científica como los conocimientos alcanzados sobre los objetos, procesos y fenómenos. Los cuales se constatan en la práctica y se corroboran a través de procedimientos tecnológicos posibilitando la comprensión de sus relaciones causales y secuenciales. Permitiendo la toma de decisiones de forma adecuada, donde se logra una mejor aplicabilidad a la situación social. Por ello la actual investigación asume esta conceptualización para la proyección metodológica, donde se promueva la renovación de las estructuras y contenidos matemáticos de acuerdo al contexto social a partir del enfoque CTS.

Para hacer realidad lo expresado por los autores antes referenciado se impone tener conocimientos basados en fundamentos científico-tecnológicos y metodológicos para abordar la formación matemática. Pero, también se necesita de una sistematización de posturas teórico-prácticas contextualizadas en la profesión, sustentadas en los procedimientos argumentativos que permitan orientar la formación matemática sistematizada a partir de los estudios CTS.

En este sentido, para minimizar las insuficiencias diagnosticadas en las carreras de perfil ingenieril antes señaladas; es imprescindible proyectar una alternativa metodológica, que propicie elevar los niveles de sistematización cognitiva de todos los estudiantes involucrados en la formación. En la cual se tenga en cuenta la precisión de López (2010), sobre la intencionalidad de proyectar acciones donde el profesor desarrolle un rol determinante en la formación del profesional. Eso presupone, la necesidad de transformar la formación matemática, a partir de la comprensión de la ciencia y técnica a través de la interacción con la sociedad. Por ello se asume en la presente pesquisa, la pertinencia de fortalecer diversos espacios formativos que permitan una participación activa de los estudiantes en la solución de los problemas. 


\subsection{La dinámica de la formación matemática sistematizada en las carreras de perfil ingenieril a partir del enfoque de Ciencia, Tecnología y Sociedad}

Se asume en la actual investigación, para la valoración de la dinámica del proceso de formación matemática sistematizada, los criterios de Álvarez de Zayas (1999); quien reconoce los componentes académico, investigativo y laboral como aspectos esenciales en el proceso formativo. Pues, estos componentes se deben integrar sustentados en el enfoque CTS. Donde se proporcione la preparación de los estudiantes como sujetos activos, capacitándolos para el desarrollo de investigaciones; a partir de aplicaciones de la ciencia y tecnología en la solución de los problemas profesionales.

Sobre este particular otros autores como: Núñez; López (2001); Pedrosa; Martins (2002); Figaredo (2003); Fontes; Cardoso (2006); Santos (2008); Mariza; Everton (2012), tienen un punto de encuentro en el abordaje de la implementación del enfoque CTS, en el proceso de formación profesional a nivel internacional. Mientras que Faustino (2014, p. 20), describió sobre la "preparación de los profesores en las ciencias y las concepciones previas del futuro ingeniero sobre la ciencia y tecnología" que ofrecen resultados claramente positivos, cuando se ponen en práctica los enfoques CTS, en los procesos investigativos de los problemas profesionales, los cuales no son suficientemente difundidos.

Asimismo, se asumen las opiniones de Faustino; Pérez, Diéguez (2014), quienes valoraron la significación que tiene la integración de los componentes académico, investigativo y laboral, los cuales incluyen la naturaleza del conocimiento de la ciencia y tecnología como una alternativa de cambio en sus relaciones con la sociedad. Por consiguiente, se reconoce así, la necesidad de trascender en la identificación de lo académico; en las formas de organización del proceso de formación matemática sistematizada. Enfocada en los estudios CTS, como una alternativa de cambio en la asimilación de los contenidos y de su puesta en práctica desde una política sólida de apoyo a la innovación tecnológica de alto nivel orientada por el MESCTI.

En este sentido, existen otros investigadores como Núñez (1999); Osorio (2002); Cachapuz (2005); Cabero (2006); Morell (2007); Bernardo; Vianna; Fontoura (2007) ; Pinheiro; Silveira Bazzo (2007) y Osorio; Martins (2011), los cuales plantean rasgos que caracterizan el enfoque CTS, lo cual puede contribuir al proceso de formación matemática sistematizada en los estudiantes de perfil ingenieril a través de las siguientes acciones:

- Usar problemas de interés local, identificados por los estudiantes, aplicando los componentes científicos-tecnológicos como organizadores del curso.

- Usar el capital humano y material, como fuentes originales de información científica que puedan emplearse en la resolución de problemas profesionales.

- Dar oportunidades para que los estudiantes desempeñen su papel de ciudadano, mientras intentan responder preguntas para identificar problemas.

En atención a las acciones planteadas anteriormente se precisa de cambios trascendentes en los planes de estudios de las instituciones públicas, público-privadas y privadas, en la República de Angola, a partir de una consideración de la formación matemática, como proceso formativo orientado a fortalecer la cultural tecnológica. Que requiere una actitud lúcida en función de los conocimientos de la ciencia y tecnología en los contextos sociales contemporáneos, para transformar la formación matemática la cual ha estado centrada en los contenidos, con un fuerte enfoque reduccionista, al no alcanzar niveles contextualizadores por medio del enfoque CTS. Por esta razón, se requiere de la generación de espacios educativos, a través de las prácticas laborales y proyecciones de acciones metodológicas adecuadas; para el perfeccionamiento de los planes de estudios a partir del enfoque CTS.

Esta labor también tendrá en cuenta las valoraciones de otros autores como Abric (2001); Acevedo (2001); Cruz (2001); Leal; Gouvêa (2001); Cerezo (2002); Auler (2002); Acevedo; Alonso Massanero 
(2004); Mortimer; Santos (2000); Osorio (2002); Pinheiro; Bazzo, (2005); Ciavatta (2006); Echeíta (2006); De Oliveira, Tiyomi; Rodrigues (2007), Pinheiro; Silveira; Bazzo (2007); León, Vallejo; Obregoso (2010); Villegas; González (2011); Faustino; Pérez; Diéguez (2014), quienes coinciden en indicar para las Instituciones Superiores, la necesidad de proporcionar a los estudiantes una visión general e integrada de los métodos, técnicas y el contexto que les permita tomar conciencia de la aplicabilidad de los conocimientos, a partir de un acercamiento entre la ciencia y tecnología en la solución de problemas profesionales.

Entonces, la formación matemática que se desea perfeccionar a partir del enfoque CTS en la actual investigación, exige una dinámica donde el proceso de enseñanza-aprendizaje adquiera mayor sistematización. Por ellos, la formación matemática se identifica como formación matemática sistematizada, la cual promueva la cultura tecnológica en los estudiantes donde se posibilite una participación activa, elevando los niveles de compromisos sociales en la medida que se desarrolle la aplicación de los conocimientos, en función de posibilitar soluciones a las problemáticas de la profesión, respondiendo a las demandas sociales.

Las necesidades sociales demandan no solo de un profesional con sólida formación científica-tecnológica, para resolver los problemas profesionales; sino que además requiere de una formación matemática sistematizada, la cual en su constante interacción entre la ciencia y la tecnología, el estudiante en su formación, pueda apropiarse del proceso de abstracción matemática científica, al realizar valoraciones y desarrollar una actitud de indagación en la búsqueda de respuestas a sus necesidades básicas.

La abstracción matemática científica en la dinámica del proceso de formación matemática sistematizada, es síntesis de la comprensión, explicación e interpretación de las necesidades sociales de un modo más esencial y profundo al considerar sus aspectos y cualidades esenciales, con una lógica para construir una representación del problema profesional, basado en la valoración de las interacciones de todos sus aspectos como expresión de la totalidad que se reconstruye y resignifica en un proceso en constante desarrollo y autodesarrollo.

El proceso de desarrollo de abstracción matemática científica, se revela desde las "abstracciones simples hasta llegar a construir sistemas teóricos complejos” (Faustino 2014, p.70). Mostrando en la práctica una superación constante en las valoraciones, lo cual permite comprender con mayor profundidad los problemas profesionales, al destacar en el objeto estudiado lo más importante y las circunstancias que dificultan la comprensión del objeto de investigación.

Por tanto, en la dinámica de la formación matemática sistematizada, las abstracciones posibilitan el desmembramiento real del problema profesional, en el objeto de investigación, en sus partes integrantes y recomposición de las partes en un todo; lo cual presupone un proceso constante de análisis-síntesis, en conocer las partes como elementos de un todo complejo, en la sistematización del conocimiento matemático. Entonces, en el proceso de formación matemática sistematizada, la comprensión y explicación de los problemas profesionales y sociales, se desarrollan mediante la propia dinámica de acercamiento al objeto de investigación mediado por la comprensión.

La formación matemática sistematizada tiene un gran significado, desde cualquier contexto donde se desarrolle la formación del profesional; cuando esta, se proyecta a partir de la abstracción matemática científica. En la cual se tengan en cuenta las informaciones seleccionadas de la situación social, mediante una proyección metodológica, permitiendo reconocer la realidad y desde ella, se facilite la aplicación de los conocimientos que se sistematizan en el componente académico. Luego profundice estos a través de la corroboración mediante la ciencia y técnica. A su vez su aplicación en la práctica laboral investigativa, siendo el espacio propicio para que los estudiantes se ubiquen en una posición de desempeño activo como actores sociales donde se ponen en práctica los conocimientos teóricos en espacios laborales reales. Realizándose diagnósticos y proyecciones de alternativas de solución a diversas problemáticas; sustentado en los métodos y procedimientos investigativos.

Por ello, los estudiantes transitan en el proceso de enseñanza-aprendizaje desde lo universal hasta lo contextual dando la posibilidad de desarrollar la formación de los profesionales, vinculados a las perspectivas contemporáneas y territoriales. Para lograr este propósito es imprescindible que los profesores y estudiantes, 
consideren la investigación como una auténtica actividad del proceso de formación del profesional, a través de la asunción de métodos de la ciencia, capaces de posibilitar la indagación e implementación de alternativas de solución.

De ahí que, elevar los niveles de sistematización cognitiva de los estudiantes, requiere del desarrollo de la comprensión de las necesidades sociales, para lograr la explicación del objeto de investigación, lo cual, permita un adecuado diagnóstico del problema profesional, acompañado de la identificación de los conocimientos teóricos (en el caso de la formación matemática), que permitan modelar una solución para la problemática, a partir de recursos tecnológicos en el procesamiento de datos y la corroboración de los posibles resultados; estos procedimientos proyectados mediante el enfoque CTS, facilitan a la vez, dentro de la dinámica, transitar desde la comprensión hasta la generalización.

Esta generalización se debe desarrollar en la formación matemática, como una generalización matemática investigativa, desde la práctica esta permite la conceptualización de variables, definiciones, teoremas, propiedades, demostraciones, axiomas y se diversifican las operaciones o cálculos en función de resolver los problemas profesionales. Así se produce un proceso de diagnóstico, modelación teórico-práctica de una alternativa de solucionar un problema determinado; para proceder a la toma de decisión en la transformación del contexto.

Justamente, la formación matemática sistematizada discurre por medio de la abstracción matemática científica y generalización matemática investigativa, como un todo que permite la ampliación de la cultura tecnológica, lo que propicia no solo en la Matemática como ciencia sino en otras de carácter sociohumanístico, el desarrollo de cualidades esenciales en los estudiantes como son: el compromiso social, profesional, su flexibilidad ante la diversidad del conocimiento humano, su trascendencia en los ámbitos de desarrollo y autodesarrollo con lo que se logra en el proceso de solución de los problemas profesionales.

El proceso de solución de los problemas profesionales se revela, como un proceso donde se desarrolla una secuencia lógica de la aplicación de métodos y técnicas de la investigación científica, desde una perspectiva que permite la ejecución de la actividad investigativa utilizando vías y procedimientos significativos para construir o resignificar el conocimiento; en aras de resolver las necesidades sociales, propiciando la formación de un profesional en el perfil ingenieril, capaz de solucionar los problemas profesionales de su contexto, aplicando un razonamiento lógico, mediante el enfoque CTS.

Por consiguiente, en el proceso de solución de problemas profesionales se van revelando vías a seguir, los métodos propios de una ciencia, técnicas de avanzada, los cuales le permitan resolver los problemas esenciales que se presentan en su campo de acción profesional de manera activa, independiente y creadora. Lo que permite fomentar su compromiso y responsabilidad con el propio desarrollo, transformación individual y se trazan nuevos retos.

Donde está presente, tanto una estructura conceptual como de procedimientos que sustentan la especificidad de la indagación; sobre el objeto de investigación en el proceso de formación matemática sistematizada, orientada al enfoque CTS. Por ende, el proceso de solución de problemas profesionales en la formación matemática sistematizada, se da en unidad entre la abstracción matemática científica y la generalización matemática investigativa (Figura1). Posibilitando un proceso de solución de problemas profesionales en función de apoyar el desarrollo económico, social y cultural. 


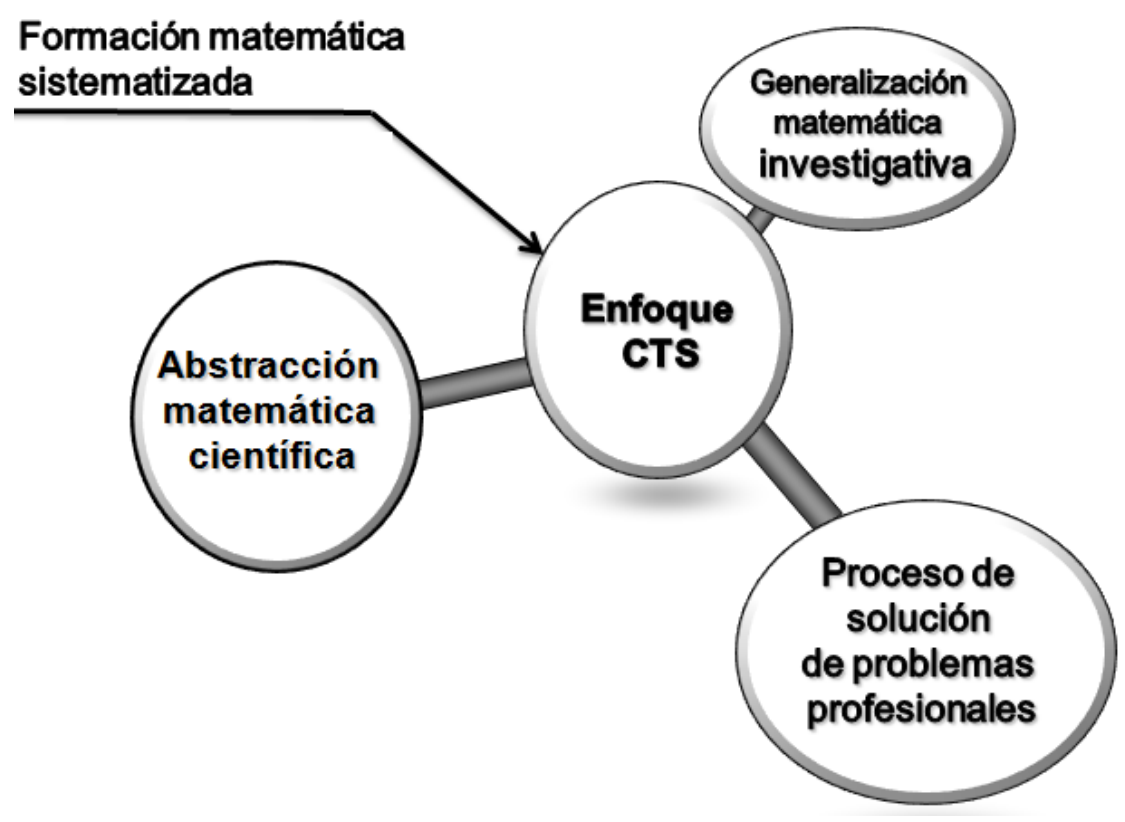

FIGURA NO.1

Formación matemática sistematizada a partir del enfoque Ciencia, Tecnología y Sociedad Elaboración propia de los autores (2014)

Por tanto, la dinámica del proceso de formación matemática sistematizada a partir del enfoque CTS, para la formación de los niveles cognitivos; se debe sistematizar, mediante la apropiación de la cultura tecnológica, a través del estrecho vínculo con el contexto, mediante acciones metodológicas las cuales se enfaticen en elevar los niveles de sistematización cognitiva de los estudiantes. Para lo cual se requiere, de un sistema de acciones metodológicas, que faciliten la ejercitación de los métodos y el uso adecuado de las tecnologías, encaminada a propiciar situaciones comunicativas en sus diferentes momentos estratégicos, a partir del nivel del desarrollo de las estructuras cognoscitivas alcanzadas, por los estudiantes en la aplicación paulatina del proceso de solución de problemas profesionales.

\section{Procedimientos metodológicos}

\subsection{Proyección de un sistema de acciones metodológicas encaminada al desarrollo de la formación matemática sistematizada, en las carreras de perfil ingenieril a partir del enfoque de Ciencia, Tecnología y Sociedad}

La aplicación de la proyección de un sistema de acciones metodológicas, en las carreras de perfil ingenieril, para el desarrollo de una formación matemática sistematizada a partir del enfoque CTS (De Armas, Lorences y Perdomo 2003), emerge de la relación entre la abstracción matemática científica y la generalización matemática investigativa, a fin de perfeccionar la superación profesoral que posibilite una elevación en los niveles de sistematización cognitiva en los estudiantes revelando relaciones establecida entre abstracciones matemáticas científicas y generalizaciones matemáticas investigativas; permitiendo solucionar problemas profesionales, mediante enfoques científicos dialécticos (Ciencia, Tecnología, Sociedad).

Las acciones metodológicas a implementarse desde carreras ingenieriles, para desarrollar una formación matemática sistematizada, están sustentadas por superaciones profesorales, mediante actividades metodológicas sistemáticas (reuniones metodológicas, clases metodológicas instructivas, clases abiertas, controles a clases, talleres socializadores). Donde se orientan, demuestran, comprueban y socializan 
indicaciones en el proceso de enseñanza-aprendizaje; como perfeccionamientos didácticos. Así, este ciclo permite preparar adecuadamente a las y los profesores (profesores), acumulando mayor experiencia pedagógica.

Además, se delimitan estructuras didácticas, que dinamizan la proyección metodológica. Seguidamente se explican las mismas a partir de una lógica metodológica, en una asignatura básica del perfil ingenieril, mediante una clase metodológica instructiva donde se definen habilidades intelectuales, docentes e investigativas; sustentadas en la formación matemática, para resolver problemas profesionales y sociales. Luego se desarrolla una ejemplificación con la presencia de algunos estudiantes seleccionados del perfil ingenieril, a partir de una actividad docente, posibilitando desde una asignatura básica de la especialidad ingenieril; la proyección de una solución a un problema determinado de una empresa pública, públicaprivada o privada con la ayuda de algoritmos, cálculos matemáticos y aplicaciones informáticas que permitan con pertinencia responder a una demanda económica social, siendo realizada dichas actividad en una clase demostrativa desarrollada en las IES.

Posteriormente, se procedió a demostrar todas las orientaciones metodológicas y didácticas en todas las acciones de una clase modelo en el perfil ingenieril, conocido como clase abierta; donde participaron los profesores como espectadores, lo cual, se analiza al finalizar, para valorar el cumplimiento de las indicaciones metodológicas a partir de este ejercicio se procede a desarrollar diversos sistemas de clases en las carreras de Ingeniería Civil, Informática, Eléctrica e Hidráulica, en lo cual se comprueba mediante el control a clase, el cumplimiento de las indicaciones metodológicas y didácticas. Así se experimentó varias formas organizativas dentro del perfil ingenieril en la las IES”, en las provincias de Bié, Benguela, Huambo, Lubango y Luanda respectivamente en la República de Angola, donde la formación matemática logra, desde el enfoque CTS elevar la sistematización cognitiva de los estudiantes, aplicando la teoría a la práctica en a solución de problemas.

Así se desarrolló diversas experiencias pedagógicas, que se socializan en un taller metodológico permitiendo el diálogo entre profesores sobre esta dinámica, que facilito delimitar nuevas insuficiencias metodológicas y didácticas, las cuales se perfeccionarán en el seguimiento metodológico del proceso de enseñanza-aprendizaje.

\subsection{Diagnóstico de la situación actual de la formación matemática en las carreras de perfil ingenieril}

Objetivo estratégico: caracterizar el proceso de enseñanza-aprendizaje de la Matemática en las carreras de perfil ingenieril.

En este proceso se realiza acciones como:

- Valorar el sistema de trabajo metodológico de las carreras de perfil ingenieril, para apreciar la proyección de acciones en función de facilitar soluciones a los problemas profesionales a partir de la formación matemática de los estudiantes.

- Identificar los enfoques empleados para la valoración de los componentes de ciencia, tecnología y sociedad en las carreras de perfil ingenieril, mediante encuestas a las y los estudiantes (estudiantes) y el claustro de las IES, de las provincias de Bié, Benguela, Huambo, Lubango y Luanda respectivamente en la República de Angola.

- Identificar los niveles de sistematización cognitiva, que se posibilitan a partir del enfoque CTS, en los programas de las disciplinas y de asignaturas en las carreras de perfil ingenieril en las IES, de las provincias de Bié, Benguela, Huambo, Lubango y Luanda respectivamente en la República de Angola.

- Realizar observaciones a clases para constatar la utilización de métodos de la ciencia y técnica en el proceso de enseñanza-aprendizaje. 
- Realizar observaciones a clases para constatar los niveles de sistematización en los estudiantes, a través de la comprensión, análisis, indagación, explicación, argumentación y aplicación de los conocimientos matemáticos a partir de la perspectiva CTS.

- Valorar la proyección metodológica de la práctica laboral investigativa donde se insertan los estudiantes.

- Valorar las proyecciones metodológicas utilizadas para el logro de la sistematización cognitiva en los estudiantes.

- Valorar los medios tecnológicos que se emplean para la corroboración de los resultados investigativos dentro de la formación matemática.

- Valorar los niveles de satisfacción de los estudiantes relacionado con el proceso de enseñanzaaprendizaje de la Matemática mediante encuestas.

Así se pueden conocer las insuficiencias presentes, en el proceso formativo de los estudiantes de las carreras de perfil ingenieril, donde las asignaturas de las especialidades ingenieriles, se sustentan en la formación matemática; sin el logro de una adecuada sistematización cognitiva. Por ello, se requiere de una orientación metodológica para los profesores encargados de desarrollar el proceso de enseñanza-aprendizaje la cual se propicie a partir del perfeccionamiento de su dinámica.

\subsection{Proceso de orientación metodológica para la formación matemática en las carreras de perfil ingenieril}

Objetivo estratégico: Orientar metodológicamente a los profesores del colectivo de la asignatura de Matemática, sobre la formación matemática sistematizada a partir del enfoque Ciencia, Tecnología y Sociedad, en el perfil ingenieril.

El logro de esta finalidad requiere de acciones como:

Establecer indicación metodológica para la proyección de acciones encaminada al desarrollo de la formación matemática sistematizada en las carreras de perfil ingenieril a partir del enfoque de Ciencia, Tecnología y Sociedad:

- Identificar las relaciones dialécticas que se establecen entre Ciencia, Tecnología y Sociedad en el contenido teórico matemático (variables, definiciones, teoremas, propiedades, demostraciones, axiomas), en el perfil ingenieril.

- Identificar los métodos matemáticos en la solución de problemas profesionales en el perfil ingenieril.

- Seleccionar los medios tecnológicos para la corroboración de los resultados investigativos dentro del perfil ingenieril.

- Desarrollar reuniones metodológicas, para indicar orientaciones sobre el desarrollo de la formación matemática sistematizada en las carreras de perfil ingenieril a partir del enfoque de Ciencia, Tecnología y Sociedad.

- Desarrollar clases metodológicas instructivas y demostrativas sobre la aplicación del enfoque de Ciencia, Tecnología y Sociedad en la formación matemática sistematizada en las carreras de perfil ingenieril.

- Desarrollar clases abiertas sobre la aplicación del enfoque de Ciencia, Tecnología y Sociedad en la formación matemática sistematizada en las carreras de perfil ingenieril, para demostrar didácticamente su proyección.

- Desarrollar controles a clases para verificar metodológicamente, la aplicación del enfoque de Ciencia, Tecnología y Sociedad en la formación matemática sistematizada en las carreras de perfil ingenieril. 
- Desarrollar un taller metodológico sobre la aplicación del enfoque de Ciencia, Tecnología y Sociedad en la formación matemática sistematizada en las carreras de perfil ingenieril, para socializar las experiencias pedagógicas logradas.

La orientación metodológica, se realizó al claustro de profesores que atienden académica y científicamente a los estudiantes, de las carreras de perfil ingenieril; sustentado en la formación matemática, donde se procede a implementar un sistema de acciones, en función de elevar los niveles de sistematización cognitiva a partir de una adecuada valoración de las problemáticas de la profesión y la sociedad, desde un enfoque CTS. Que facilita una visión acertada del contexto, las demandas sociales y las perspectivas económicas-medio ambientales.

\subsection{Solución de problemas profesionales o sociales a través de la formación matemática sistematizada de los estudiantes de perfil ingenieril}

Objetivo estratégico: elevar los niveles de sistematización cognitiva de los estudiantes de perfil ingenieril, en el proceso de solución de problemas profesionales y sociales. Utilizando para ellos los conocimientos, métodos y técnicas de investigación científica, en la dinamización de la formación matemática sistematizada, como síntesis de la abstracción matemática científica y generalización matemática investigativa.

En este proceso se pueden realizar las siguientes acciones:

- Identificar los problemas profesionales o sociales que requieran investigación científica para su solución en el perfil ingenieril.

- Identificar los contenidos matemáticos necesarios, para solucionar los problemas profesionales o sociales determinados en el perfil ingenieril.

- Modelar una alternativa de solución a un problema profesional o social, a partir del empleo de recursos tecnológicos; aplicando métodos y técnicas de investigación científica en la formación matemática sistematizada en el perfil ingenieril.

- Demostrar la veracidad de un problema profesional o social del perfil ingenieril mediante la aplicación de los asistentes matemáticos.

- Demostrar la viabilidad de una alternativa de solución, a un problema profesional o social, mediante el desarrollo de los procesos de deducción-inducción, analogía-modelación; presentes en la abstracción del problema en el perfil ingenieril.

- Argumentar en el proceso de solución de problemas profesionales o sociales, la significatividad de la formación matemática sistematizada; a través de la integración de los componentes de Ciencia, Tecnología y Sociedad, como elementos imprescindibles para un enfoque pertinente.

- Valorar la importancia de la integración de los conocimientos matemáticos y los recursos tecnológicos en la indagación de las demandas sociales.

- Valorar la repercusión en el contexto, del proceso de solución de los problemas profesionales o sociales; logrado a partir de la formación matemática sistematizada.

La solución de problemas profesionales o sociales, a través de la formación matemática sistematizada, se realizará de forma flexible teniendo en cuenta el seguimiento continuo del diagnóstico, el cual se logra a través de un sistema de evaluación, a las acciones metodológicas desarrolladas en el proceso formativo de las carreras de perfil ingenieril. Donde se aprecian las manifestaciones de las insuficiencias académicas e investigativas de los estudiantes; así como también el rol conductor de los profesores. 


\subsection{Evaluación de las acciones metodológicas empleadas para el desarrollo de la formación matemática sistematizada en las carreras de perfil ingenieril}

Objetivo estratégico: valorar los resultados de la implementación del sistema de acciones metodológicas, para la formación matemática sistematizada en las carreras de perfil ingenieril.

\section{Indicadores para la evaluación del desarrollo de la formación matemática sistematizada en las carreras de perfil ingenieril}

En este proceso se determinó los siguientes indicadores:

- Exigencias académicas-investigativas para la formación matemática sistematizada en las carreras de perfil ingenieril.

- Principios necesarios para el desarrollo de la formación matemática sistematizada, a partir del enfoque CTS en las carreras de perfil ingenieril.

- Indicaciones metodológicas para el desarrollo de la sistematización cognitiva en los estudiantes de las carreras de perfil ingenieril.

\section{Indicadores para la evaluación de los niveles alcanzados en el proceso de sistematización cognitiva en las carreras de perfil ingenieril}

En este proceso se determinó los siguientes indicadores:

- Automatización de los procesos de deducción-inducción y analogía-modelación; presentes en la abstracción del problema profesional o social en el perfil ingenieril.

- Procesamiento de datos en la solución de problemas profesionales o sociales, mediante una adecuada clasificación, a partir del enfoque CTS en las carreras de perfil ingenieril.

- Cálculo matemático sustentado en la adecuada selección de métodos y técnicas de investigación científica en la solución de los problemas profesionales o sociales en las carreras de perfil ingenieril.

- Constatación de las transformaciones logradas en el contexto, a partir de la comprobación del proceso de solución de los problemas profesionales o sociales, sustentados en la formación matemática sistematizada.

La delimitación de indicadores que posibiliten la evaluación de las transformaciones en el proceso formativo de los estudiantes de las carreras del perfil ingenieril, se desarrolló partiendo del diagnóstico de las insuficiencias en el proceso de enseñanza-aprendizaje. Lo cual posibilita una apreciación de los componentes necesarios, para modificar los desempeños de los profesores de las carreras del perfil ingenieril en el desarrollo de actividades académicas e investigativas, teniendo en cuenta los siguientes aspectos a evaluar:

- Preparación metodológica que poseen los profesores del colectivo de Matemática, para el desarrollo de los niveles de sistematización cognitiva; a partir del enfoque CTS en los estudiantes de las carreras del perfil ingenieril.

- Desarrollo del sistema de acciones metodológicas realizadas con el claustro de profesores de las carreras del perfil ingenieril.

- Valorar los resultados alcanzados en el proceso formativo de las carreras de perfil ingenieril, a partir de la sistematización de los conocimientos de Álgebra Lineal, Matemática Numérica y Geometría Analítica a partir del enfoque Ciencia, Tecnología y Sociedad. 
- Valorar los niveles de satisfacción alcanzados por profesores y estudiantes en cuanto a las acciones metodológicas implementadas a partir de la formación matemática sistematizada. Se adjunta la tabla 1.

TABLA 1

Niveles de satisfacción en el perfil ingenieril, en cuanto a las acciones metodológicas para la formación matemática sistematizada

\begin{tabular}{|l|l|l|}
\hline \multirow{2}{*}{ CRITERIOS VALORATIVOS } & \multicolumn{2}{|l|}{$\begin{array}{l}\text { PORCIENTO DE } \\
\text { SATISFACCION }\end{array}$} \\
\cline { 2 - 3 } & \multicolumn{2}{|l|}{ CURSOS } \\
\cline { 2 - 3 } & $2013-2014$ & $2014-2015$ \\
\hline $\begin{array}{l}\text { Cumplimientos del objetivo propuesto } \\
\text { en cada proceso, en el per fil ingenieril. }\end{array}$ & $32 \%$ & $93 \%$ \\
\hline $\begin{array}{l}\text { Resistencia al cambio metodológico en } \\
\text { los profesores del per fil ingenieril. }\end{array}$ & $89 \%$ & $5 \%$ \\
\hline $\begin{array}{l}\text { Adaptación al sistema de acciones } \\
\text { metodológicas por los profesores. }\end{array}$ & $31 \%$ & $90 \%$ \\
\hline $\begin{array}{l}\text { Contribuciones al desarrollo de los } \\
\text { niveles de sistematización } \\
\text { cognoscitiva de los estudiantes. }\end{array}$ & $20 \%$ & $94 \%$ \\
\hline $\begin{array}{l}\text { El desarrollo de actividades } \\
\text { académicas-investigativas } \\
\text { Sustentadas en la formación } \\
\text { matemática sistematizada. }\end{array}$ & $45 \%$ & $92 \%$ \\
\hline $\begin{array}{l}\text { Se viabilizan las relaciones entre } \\
\text { estudiantes y el contexto. }\end{array}$ & $61 \%$ & $89 \%$ \\
\hline $\begin{array}{l}\text { Facilita las relaciones entre } \\
\text { estudiantes y sectores productivos. }\end{array}$ & $80 \%$ & $95 \%$ \\
\hline $\begin{array}{l}\text { Dinamiza la identificación con el } \\
\text { entono sociocultural. }\end{array}$ & $71 \%$ & $90 \%$ \\
\hline
\end{tabular}

Elaboración propia de los autores (2016).

\section{ANÁLISIS Y DISCUSIÓN DE LOS RESULTADOS}

\subsection{Valoración de la aplicación parcial de la formación matemática sistematizada a partir del enfoque Ciencia, Tecnología y Sociedad, en el perfil ingenieril}

Esta evaluación de la aplicación parcial del sistema de acciones metodológicas, encaminada al perfeccionamiento de la formación matemática sistematizada a partir del enfoque CTS; tuvo como sujetos en las IES, de las provincias de Bié, Benguela, Huambo, Lubango y Luanda respectivamente en la República de Angola, en la provincia de Luanda, respectivamente de la República de Angola.

En el tercer y cuarto año académico de las carreras de Ingeniería Civil, Informática, Electrónica, Telecomunicación e Información; quienes fueron sometidos a una prueba pedagógica, encuestas de satisfacción y observación participante integrada por 50 estudiantes del tercer año y 20 profesores de la carrera de Ingeniería Civil, Informática, Electrónica, Telecomunicación e Información, seleccionados intencionalmente en investigaciones realizadas desde 2013 hasta 2018, con la finalidad de identificar las transformaciones experimentadas en el proceso de formación profesional. 


\section{Análisis cuantitativo y cualitativo de los resultados de la prueba pedagógica:}

Con el fin de constatar las limitaciones académicas - investigativas de los estudiantes de las carreras de perfil ingenieril; se aplicaron pruebas pedagógicas en el tercer año del curso regular diurno desde 2013-2015, en las asignaturas básicas de la espacialidad ingenieril, donde se diagnosticarán los conocimientos sobre Álgebra Lineal, Matemática Numérica y Geometría Analítica. Así mismo sobre la delimitación de los componentes de Ciencia, Tecnología y Sociedad que aprecian en su proceso de enseñanza-aprendizaje.

Esta apreciación permitió además realizar una caracterización del grupo e identificar las necesidades e intereses de los estudiantes en la evaluación de los conocimientos antes mencionadas, los estudiantes presentaron insuficiencia en: abstraer-generalizar, demostrar-aplicar conocimientos matemáticos (principios, leyes, teoremas, axiomas y propiedades), modelar e interpretar los problemas (Tabla2).

TABLA2

\section{Valoración cognitiva en la formación matemática sistematizada del perfil ingenieril}

\begin{tabular}{|c|c|c|}
\hline \multicolumn{3}{|c|}{ Resultados del curso regular diurno 2013-2015 } \\
\hline Aspectos cognitivos & \begin{tabular}{|l|} 
Evaluación \\
Cuantitativa
\end{tabular} & $\begin{array}{l}\text { Evaluación } \\
\text { Cualitativa }\end{array}$ \\
\hline $\begin{array}{l}\text { Identificar los problemas profesionales o } \\
\text { sociales vinculados con el perfil } \\
\text { ingenieril. }\end{array}$ & $32 \%$ & Regular \\
\hline $\begin{array}{l}\text { Demostrar la veracidad de un problema } \\
\text { profesional o social del perfil ingenieril } \\
\text { mediante la aplicación de los asistentes } \\
\text { matemáticos. }\end{array}$ & $4 \%$ & Mala \\
\hline $\begin{array}{l}\text { Indagar los contenidos matemáticos } \\
\text { necesarios, para solucionar los } \\
\text { problemas profesionales o sociales } \\
\text { determinados en el perfil ingenieril. }\end{array}$ & 30 & Regular \\
\hline $\begin{array}{l}\text { Modelar una alternativa de solución a un } \\
\text { problema profesional o social, a partir } \\
\text { del empleo de recursos tecnológicos. }\end{array}$ & $14 \%$ & Mala \\
\hline $\begin{array}{l}\text { Generalizar los procesos de } \\
\text { deducción-inducción, } \\
\text { analogia-modelación; presentes en la } \\
\text { abstracción del problema en el perfil } \\
\text { ingenieril en las asignaturas básicas. }\end{array}$ & $21 \%$ & Regular \\
\hline $\begin{array}{l}\text { Seleccionar métodos y técnicas de } \\
\text { investigación para la apreciación desde } \\
\text { un adecuado enfoque CTS. }\end{array}$ & $10 \%$ & Mala \\
\hline $\begin{array}{l}\text { Aplicar los conocimientos matemáticos y } \\
\text { los recursos tecnológicos en la } \\
\text { indagación de las demandas sociales. }\end{array}$ & $10 \%$ & Mala \\
\hline $\begin{array}{l}\text { Argumentar la significatividad de la } \\
\text { formación matemática sistematizada en } \\
\text { el proceso de solución de problemas } \\
\text { profesionales o sociales. }\end{array}$ & $17 \%$ & Mala \\
\hline $\begin{array}{l}\text { Interpretar la repercusión en el contexto, } \\
\text { del proceso de solución de los problemas } \\
\text { profesionales o sociales. }\end{array}$ & $13 \%$ & Mala \\
\hline
\end{tabular}

Elaboración propia de los autores (2014).

El diagnóstico del curso regular diurno 2013-2015, reveló la necesidad de sistematizar la formación matemática a partir del enfoque Ciencia, Tecnología y Sociedad, en el perfil ingenieril. Por tanto, para contribuir a minimizar las insuficiencias antes planteadas, se implementaron acciones metodológicas al 
proceso de enseñanza-aprendizaje de los estudiantes del curso diurno, cuarto año, 2016-2018 en su estado de evolución (Tabla3). Las acciones fueron dirigidas, conscientemente, al desarrollo de la abstracción, generalización, demostración, modelación, interpretación, indagación, argumentación y aplicación de conocimientos matemáticos. Como: principios, leyes, teoremas, axiomas y propiedades en la solución de problemas matemáticos vinculados a los problemas profesionales y sociales, lo cual permitió a los estudiantes una sistematización en cognitiva, logrando por medio de métodos, técnicas de investigación, en la solución de los problemas profesionales o sociales a través de la investigación científica.

TABLA3

\section{Valoración cognitiva en la formación matemática sistematizada del perfil ingenieril}

\begin{tabular}{|l|l|l|}
\hline \multicolumn{2}{|l|}{ Resultados del curso regular diurno 2016-2018 } \\
\hline Aspectos cognitivos & $\begin{array}{l}\text { Evaluación } \\
\text { Cuantitativa }\end{array}$ & $\begin{array}{l}\text { Evaluación } \\
\text { Cualitativa }\end{array}$ \\
\hline $\begin{array}{l}\text { Identificar los problemas profesionales o } \\
\text { sociales vinculados con el perfil } \\
\text { ingenieril. }\end{array}$ & $95 \%$ & Excelente \\
\hline $\begin{array}{l}\text { Demostrar la veracidad de un problema } \\
\text { profesional o social del per fil ingenieril } \\
\text { mediante la aplicación de los asistentes } \\
\text { matemáticos. }\end{array}$ & $88 \%$ & Bien \\
\hline $\begin{array}{l}\text { Indagar los contenidos matemáticos } \\
\text { necesarios, para solucionar los } \\
\text { problemas profesionales o sociales } \\
\text { determinados en el per fil ingenieril. }\end{array}$ & $90 \%$ & Excelente \\
\hline $\begin{array}{l}\text { Modelar una alternativa de solución a un } \\
\text { problema profesional o social, a partir } \\
\text { del empleo de recursos tecnológicos. }\end{array}$ & $88 \%$ & Bien \\
\hline $\begin{array}{l}\text { Generalizar los procesos de } \\
\text { deducción-inducción, } \\
\text { analogía-modelación; presentes en la } \\
\text { abstracción del problema en el perfil } \\
\text { ingenieril en las asignaturas básicas. }\end{array}$ & $88 \%$ & Bien \\
\hline $\begin{array}{l}\text { Seleccionar métodos y técnicas de } \\
\text { investigación para la apreciación desde } \\
\text { un adecuado enfoque CTs. }\end{array}$ & $90 \%$ & Excelente \\
\hline $\begin{array}{l}\text { Aplicar los conocimientos matemáticos y } \\
\text { los recursos tecnológicos en la } \\
\text { indagación de las demandas sociales. }\end{array}$ & $88 \%$ & Excelente \\
\hline $\begin{array}{l}\text { Argumentar la significatividad de la } \\
\text { formación matemática sistematizada en } \\
\text { el proceso de solución de problemas } \\
\text { profesionales o sociales. }\end{array}$ & $90 \%$ \\
\hline $\begin{array}{l}\text { Interpretar la repercusión en el contexto, } \\
\text { del proceso de solución de los problemas } \\
\text { profesionales o sociales. }\end{array}$ & $90 \%$ & \\
\hline
\end{tabular}

Elaboración propia de los autores (2018).

\section{Análisis cuanti-cualitativo de los resultados de la encuesta de satisfacción de los estudiantes de perfil ingenieril:}

Esta valoración cognitiva, es expresión del proceso de enseñanza-aprendizaje de las carreras de perfil ingenieril, donde se tuvo en cuenta además, los niveles de satisfacción de los estudiantes, en el año 2016, los estudiantes fueron encuestados, sobre los niveles de seguridad que poseían en cuanto a la utilidad cognitiva, que revestía la formación matemática sistematizada para el desarrollo de las asignaturas básicas y solo el 
31\% reconocía los beneficios de esta (Gráfica1). Mientras solo el $43 \%$ declaró que estaban motivados a aplicar los conocimientos matemáticos en la solución de los problemas profesionales o sociales. Así mismo se manifestó en forma limitada la satisfacción de poder resolver mediante los conocimientos matemáticos desde un enfoque CTS, las problemáticas de la profesión o la sociedad, siendo solo el $24 \%$ de los estudiantes quienes lograban este proceder. Estas escalas tienen una valoración cualitativa determinada por adecuado, poco adecuada y no adecuada, los cuales manifiestan porcientos muy bajos.

Entonces se comenzó a realizar la implementación del sistema de acciones metodológicas para revertir esta situación. Por ello en el año 2018, se apreciaron transformaciones en el proceso de enseñanza-aprendizaje. Ahora las escalas de adecuada, poco adecuada y no adecuada tienen una valoración cualitativa superior los cuales manifiestan porcientos altos. Ejemplo de ello es el 69\% de los estudiantes encuestados declaran que los profesores utilizan el contenido de la Matemática en la familiarización de los problemas profesionales o sociales de forma adecuada, a través del uso de la proyección de acciones metodológicas encaminada al desarrollo de la formación matemática sistematizada enfocada en los estudios CTS. Mientras todavía el $34 \%$ de los estudiantes alegan que la vinculación de los problemas matemáticos enfocados en los estudios CTS es poco visible, siendo esta poco adecuada la proyección metodológica. Así mismo otros estudiantes que representan el 20\%, manifiestan que algunos profesores lo realizan, pero de forma insuficiente, siendo valorado de no adecuado (Gráfica1).

Desde el año 2016 hasta 2018, donde ya se ha realizado la proyección metodológica; se aprecia una evolución de un 76\% al 92\% de satisfacción de los estudiantes hacia la formación matemática sistematizada, en la solución de los problemas profesionales o sociales, siguiendo la lógica de la investigación científica. Sin embargo, la utilización de métodos, técnicas y contenidos vinculados directamente al problema profesional o social a resolver por el estudiante se manifiesta con una leve mejoría de un $41 \%$ al $59 \%$, por lo que se determina como poco adecuado. (Gráfica2). Mientras que solo del 19\% al 7\% de los estudiantes revelan tener algunas imprecisiones en la argumentación de los problemas profesionales o sociales (Gráfica2).
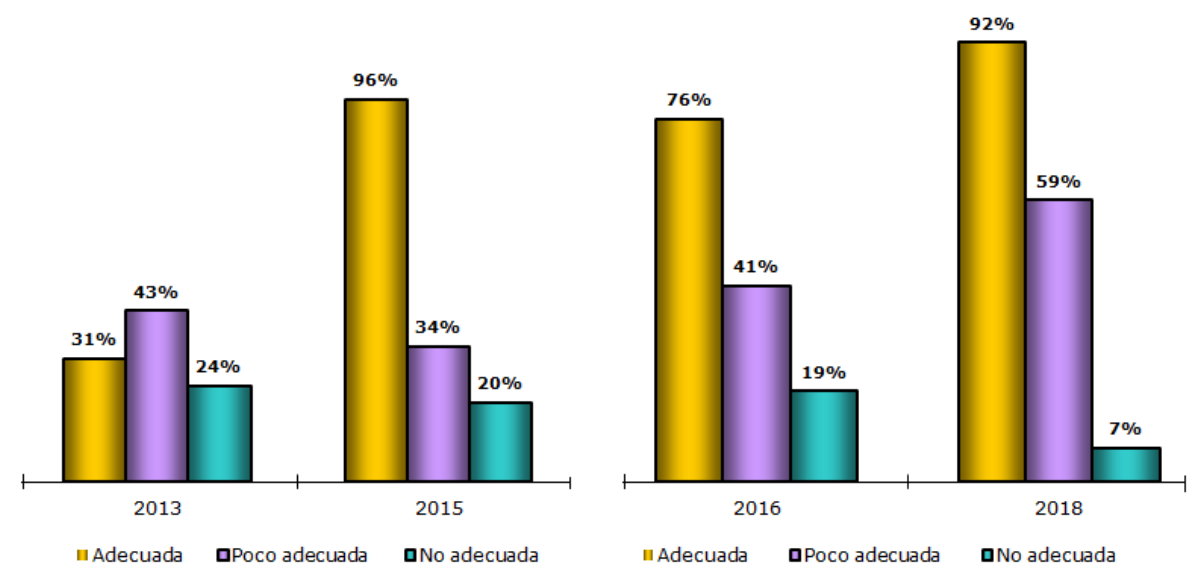

Gráfica No.1: Resultados del pre y postest(2013-2015)

Gráfica No.2: Resultados del pre y postest(2016-2018)

Elaboraciones propias de los autores (2018)

\section{Análisis de la observación participante de los niveles de sistematización cognitiva}

Durante la implementación de acciones metodológicas, en el desarrollo académico-investigativo de las asignaturas básicas de las carreras de perfil ingenieril. Se pudo apreciar las siguientes transformaciones en el proceso de enseñanza-aprendizaje; mediante la sistematización cognitiva de los conocimientos de Álgebra Lineal, Matemática Numérica y Geometría Analítica. En el cuarto año en las IES, en las provincias de Bié, 
Benguela, Huambo, Lubango y Luanda respectivamente en la República de Angola. En el tercer y cuarto año académico de las carreras de Ingeniería Civil, Informática, Electrónica, Telecomunicación e Información.

- Se aprecia un incremento notable en la utilización de métodos de la ciencia y la técnica, los cuales permiten vincular los contenidos matemáticos directamente al problema profesional o social a resolver por el estudiante.

- Se emplean en el desarrollo de las clases el uso de recursos para desarrollar habilidades de indagación, explicación y argumentación, a través de la confrontación de diferentes teorías, que permiten que el estudiante realice sus propias valoraciones en el contexto de la asignatura y de la profesión.

- En los estudiantes se evidencia el desarrollo de las habilidades para identificar situaciones problémicas en el marco de la profesión, asignaturas o la sociedad.

- Se identifican y fundamentan los problemas profesionales o sociales de forma coherente.

- Los estudiantes emiten juicios valorativos sobre los resultados obtenidos, todo lo cual le permitió llevar a feliz término la presente investigación.

Una vez finalizada la aplicación parcial, de la proyección del sistema de acciones metodológicas, en el año 2018, para el perfeccionamiento de la formación matemática sistematizada a partir del enfoque CTS. Se procederá a una evaluación integral de todos los procesos para realizar un informe resumen, donde delimiten las deficiencias detectadas en la argumentación de los problemas profesionales o sociales. Para su erradicación mediante nuevas propuestas metodológicas.

\subsection{Constatación cientifica de los resultados investigativos}

Para la efectividad de la constatación de los resultados científicos, fueron realizadas quince visitas a actividades docentes, en las que se valoró la implementación de las orientaciones metodológicas. Esta apreciación fue comparada con el diagnóstico inicial, a través de una prueba de hipótesis de los rangos con signo de Wilcoxon, para dos muestras relacionadas.

La metodología aplicada para desarrollar las visitas a actividades clase se asumió el paradigma cualicuantitativo de investigación, a través de la aplicación de las pruebas pedagógica, la triangulación de los datos y la técnica de muestreo aleatorio estatificado, como estrategia de diseño de la investigación, fue posible ajustar en las disciplinas de Álgebra Lineal, Matemática Numérica y Geometría Analítica, con amplia aplicación en el campo educativo (Hernández, Fernández y Baptista, 2006). En las visitas a clases se aplicó la técnica grupal desde talleres de socialización donde en 3 fases: pre activa, activa y pos activa se desarrolló la actividad metodológica. En la fase pre activa el propósito fue provocar en el contexto universitario debates y reflexiones que condujeran a un diagnóstico del proceso de formación matemática y comenzar la sistematización de un marco teórico referencial que diera el acceso a una brecha epistemológica de la Matemática enfocadas en el eje Ciencia, Tecnología y Sociedad.

Los resultados de las actividades metodológicas fueron prefijados desde el valor del nivel de significación en $\alpha=0,05$ y se realizó el procesamiento estadístico de la información haciendo uso del software IBMSPSS Statistics 20, para el procesamiento de la información, obteniéndose que la Significación Asintótica (Sig.) es igual a cero. Por ende, al ser la $\operatorname{Sig}<\alpha$, se puede concluir que la diferencia observada entre la prueba pedagógica realizada antes de la proyección metodológica y después de haber recibido esta formación desde los conocimientos de Álgebra Lineal, Matemática Numérica y Geometría Analítica, es significativa. Todo lo anterior permitió entonces, corroborar la validez y pertinencia de la proyección de un sistema de acciones metodológicas, al contribuir al perfeccionamiento de la dinámica de formación matemática sistematizada a partir del enfoque CTS en los estudiantes de las carreras de perfil ingenieril. 


\section{Conclusiones}

La presente investigación de carácter metodológico, se desarrolló a partir del perfeccionamiento de la dinámica del proceso de formación matemática enfocada en el eje Ciencia, Tecnología y Sociedad, con la intencionalidad de lograr la sistematización cognitiva, donde se revelaron las siguientes resultantes:

- Haber revelado las tendencias históricas del proceso de formación matemática sistematizada a partir del enfoque Ciencia, Tecnología y Sociedad en las Instituciones de Educación Superior en la República de Angola, en correspondencia con la proyección de un sistema de acciones metodológicas determinados en cada etapa, avizoran el papel de la universidad en la solución de los problemas del país, que no logran la pertinencia social requerida debido a que aún persisten insuficiencias en el desarrollo de la capacidad y la voluntad transformadora de los profesores para intervenir en los problemas del contexto o sociales.

- La proyección de un sistema de acciones metodológicas para la dinámica del proceso enseñanzaaprendizaje de la Matemática posibilita el desarrollo del razonamiento lógico en los estudiantes, mediante acciones que se organizan en los niveles de sistematización cognitiva, sustentado en una relación establecida entre la abstracción matemática científica y la generalización matemática investigativa; en correspondencia con las dimensiones y relaciones fundamentales reveladas en la modelación de los problemas sociales.

- La consulta realizada a los profesores y estudiantes encuestados sobre la temática, permitió realizar valoraciones sobre, la dinámica del proceso enseñanza-aprendizaje de la Matemática y la proyección de un sistema de acciones metodológicas para el desarrollo de este proceso, existiendo consenso en cuanto a factibilidad y pertinencia de ambos aportes, lo que posibilitó la introducción parcial de la estrategia en las carreras de Ingeniería Civil, Informática, Electrónica, Telecomunicación e Información, Informática de la en las IES angoleñas.

- Los estudiantes de las carreras de Ingeniería Civil, Informática, Electrónica, Telecomunicación e Información, de las IES”, en las provincias de Bié, Benguela, Huambo, Lubango y Luanda, respectivamente en la República de Angola, consideraron, que la formación matemática sistematizada es una forma viable, para superar las dificultades cognitivas, presentes en el proceso de enseñanza-aprendizaje de las asignaturas básicas de la espacialidad de ingeniería.

- Se permitió la sistematización cognitiva en las asignaturas básicas de las carreras de perfil ingenieril, a partir del enfoque CTS.

- Se logra una transformación social significativa en el contexto, donde los estudiantes de las carreras de Ingeniería Civil, Informática, Electrónica, Telecomunicación e Información, de las IES”, en las provincias de Bié, Benguela, Huambo, Lubango y Luanda respectivamente en la República de Angola, son orientados a solucionaren los problemas profesionales en la práctica laboral investigativa a partir de un adecuado enfoque de Ciencia, Tecnología y Sociedad. Todo lo cual demuestra el cumplimiento del objetivo de la pesquisa.

\section{A MOdo DE SUgerencias}

La proyección del sistema de acciones metodológicas encaminada al desarrollo de la formación matemática sistematizada a partir del enfoque CTS; puede ser aplicada definitivamente en la Educación Superior Angoleña. Mediante acciones que dinamizan los procesos propuestos anteriormente, los cuales conducen al logro de los objetivos planteados para solución de las necesidades sociales, haciendo uso adecuado de los conocimientos matemáticos, técnica, métodos de investigación científica. Así en aras de contribuir al perfeccionamiento de la presente pesquisa, el colectivo de autores de la presente investigación, recomienda, 
realizar indagaciones relacionadas con el diseño del proceso de formación matemática sistematizada, en las carreras de perfil ingenieril.

\section{ReFERENCIAS}

Abric, J. (2001). Prácticas sociales y representaciones. México: Ediciones Coyoacán.

Acevedo, D., Vázquez, A. y Manassero, M (2003): Papel de la educación CTS en una alfabetización científica para todas las personas. Revista Electrónica de Enseñanza de las Ciencias, 2,( 2) , pp. 1-24.

Acevedo, J. (2001) La formación del professora do de enseñanza secundarias para la educación CTS: una cuestión problemática. Revista Ibero-Americana de Educação 2 (2), pp.133-162.

Acevedo, J., Alonso, Á. y Massanero, M. (2004) Progresos en la evaluación de actitudes relacionadas con la ciencia mediante el cuestionario de opiniones CTS. Revista Ibero-Americana de Educação 4 (2), pp.136-167.

Álvarez de Zayas, C. (1999). La escuela en la vida. La Habana: Editorial Pueblo y Educación.

Auler, D. (2002). Interações entre Ciência-Tecnologia-Sociedade no contexto da formação de professores de ciências. Florianópolis, (Tese de Doutorado em Educação: Ensino de Ciências sem publicar) - Universidade Federal de Santa Catarina, Brasil.

Beckett, P. y Margutti do Amaral, G. (2005). La lectura de un texto como estratégia de investigación del pensamiento CTS: las visiones de los futuros profesores de biología. Revista Eureka sobre Enseñanza y Divulgación de las Ciencias, 2, (2), pp. 141-154.

Bernardo, J., Vianna, D. y Fontoura, H. (2007). A Produção e consumo de energia elétrica: a construção de uma proposta baseada no enfoque Ciência-Tecnologia-Sociedade-Ambiente (CTSA). Buenos Aires, pp 1-12.

Cabero, J. (2006). Bases pedagógicas del e-learning. Revista de Universidad y Sociedad del Conocimiento, 3(1), 1-10.

Cachapuz, A. (2005). Ciência-Tecnologia-Sociedade: Um compromisso ético. Revista Iberoamericana de Ciencia, Tecnología y Sociedad, 2 (6), pp.173-194.

Cerezo, J. (2002). Ciência, Tecnologia e Sociedade: o estado da arte na Europa e nos Estados Unidos. In: SANTOS, L. W. (Org.). Ciência, tecnologia e sociedade: o desafio da interação. Revista Londrina: 4 (2) pp.3-38.

Chaves, J. (2004). Desarrollo tecnológico en la primera Revolución Industrial. Revista de Historia (17), 93-109.

Ciavatta, M. (2006). Os Centros Federais de Educação Tecnológica e o ensino superior: duas lógicas em confronto. Revista Educação e Sociedade, Campinas, 27, (9)6 - Especial, pp.911-934. Disponível em: . Acesso em:05 maio 2011

Comênio, J. A. (1966). Didactica Magna: Tratado da arte Universal de ensinar tudo a todos. Lisboa pp.133-162.

Cruz, S. (2001); Aprendizagem centrada em eventos: uma experiência com enfoque Ciência, Tecnologia e Sociedade no Ensino Fundamental. Florianópolis, (Tese de Doutorado em Educação: Ensino de Ciências sem publicar) Centro de Educação, Universidade Federal de Santa Catarina, Brasil.

Cuicas, C., Casadei, C.y Álvarez V. (2007). El software matemático como herramienta para el desarrollo de habilidades del pensamiento y mejoramiento del aprendizaje de las matemáticas. Revista Actualidades Investigativas en Educación, 7(2), 1-34. DOI http://dx.doi.org/10.15517/aie.v7i2.9264

De Armas, N., Lorences, J. y Perdomo, J. (2003). Caracterización y diseño de los resultados científicos como aportes de la investigación educativa. Trabajo presentado en el curso 85 realizado en el Evento Internacional Pedagogía 2003. La Habana, Cuba.

De Oliveira, L., Tiyomi, A. y Rodrigues, A. (2007). Educação ambiental: concepções e práticas de professores de ciências do ensino fundamental. Revista Electrónica de Enseñanza de las Ciencias, 6(3), 471-495.

Echeíta, G. (2006) Educación para la inclusión. Educación sin exclusiones Madrid: Narcea.

Faustino, A. (2014). La formación del pensamiento matemático-investigativo en los estudiantes de la carrera de Licenciatura en Matemática [Tesis en opción al grado científico de Doctor en Ciencias Pedagógicas] Centro de Estudios Manuel F. Gran, Santiago de Cuba. 
Faustino, A., Pérez, N. y Diéguez R. (2012). Propuesta didáctica para el proceso de formación del pensamiento lógico matemático complejo en la educación superior angolana. Revista Pedagogía profesional 10(4), 01-10 Recuperado de http://www.pedagogiaprofesional.rimed.cu

Faustino, A. Pérez, N. y Diéguez, R. (2014). El pensamiento matemático-investigativo desde el enfoque científico tecnológico. Revista Multiciencias, vol. 14, núm. (1), 2014, pp. 80-87. Recuperado de http://www.redalyc.org/ articulo.oa?id=90430816011

Figaredo, F, (2003): Fines de la educación CTS en Cuba. (Tese de Doutorado em Educação: Ensino de Ciências sem publicar); Cuba.

Fontes, A. y Cardoso, A. (2006): Formação de professores de acordo com a abordagem Ciência/Tecnologia/Sociedade. Revista Electrónica de Enseñanza de las Ciencias 5,(1), pp. 15-30.

Fuentes, H. (2009). Pedagogía y Didáctica de la Educación Superior. [Versión digital pdf]. Recuperado de: http:// www.utelvt.edu.ec/DOCTORADO\%20PHD/TEXTOS\%20IND/P\%20E\%20S\%2009\%20.pdf

Fuentes, H., Matos, E. y Cruz S. (2004). La formación de investigadores desde pensamiento hermenéutico-dialéctico. Santiago de Cuba: Centro de Estudios de la Educación Superior "Manuel F. Gran”, Universidad de Oriente, Santiago de Cuba, Cuba pp 1-12.

García, V. y Antonio, B. (2001). Desigualdades sociales, nuevas tecnologías y política educativa. En Manuel Area (coord.), Educar en la sociedad de la información (pp. 179-213). Bilbao: Declée de Brouwer.

Gómez, G. y Mateos, B. (2002). Retos educativos en la sociedad de la información y la comunicación. Revista latinoamericana de tecnología educativa, 1(1), pp. 9-22.

Guzmán, A. (2008). Estudiantes universitarios: entre la brecha digital y el aprendizaje. Revista Apertura, 8(8), pp. 21-33.

Hernández R, Fernández, C. y Baptista, L (2006). Metodología de la investigación (4a. ed.). México: Editorial McGraw-Hill.

Leal, M. y Gouvêa, G.(2001); Uma visão comparada do ensino em ciência, tecnologia e sociedade na escola e em um museu de ciência. Revista Ciência \& Educação, Bauru, 7 (1), pp.67-84.

León, J., Vallejo, C., Parra, A. y Obregoso, Y. (2010). Clasificación múltiple de ítems y las representaciones sociales sobre ambiente en profesores rurales. Revista Electrónica, Actualidades investigativas en educación, 10(2), 1-26. Recuperado de http://revista.inie.ucr.ac.cr/uploads/tx_magazine/items.pdf

López, F. (2010). Componentes para la estructura didáctica de un curso de Educación a Distancia usando como herramienta las plataformas gestoras. (Tesis en opción al grado científico de Doctor en Ciencias Pedagógicas sin publicar) Universidad Pedagógica de Cienfuegos “Conrado Benítez García”, Cuba.

Mariza, R., Everton B. (2012) Educação tecnológica: Uma Nova Perspectiva Pedagógica. Revista Electrónica Actualidades investigativas en educación,12, (3) pp. 1-25. Recuperado de https://www.redalyc.org/ $\mathrm{html} / 447 / 44723985006 /$

Morell, D. (2007), Formación del Profesorado de Ciencias Agronómicas de la Universidad cubana de Ciego de Ávila en Educación Ciencia-Tecnología-Sociedad. (Tesis para optar por el grado de Doctorado en Ciencias Pedagógicas, sin publicar). Universidad de Granada, España.

Mortimer, E. y Santos, W. (2000). Uma análise de pressupostos teóricos da abordagem C-T-S (Ciência-TecnologiaSociedade) no contexto da educação brasileira. Ensaio -Pesquisa em Educação em Ciências, Revista Belo Horizonte, 2, (2), pp.133-162

Núñez, J, y López, C. (2001): Innovación tecnológica, innovación social y estudios CTS en Cuba. En Desafíos y tensiones actuales en Ciencia, tecnología y Sociedad. Madrid, Editorial Biblioteca Nueva

Núñez, J., Ortega C., Mauro, L., Passailaigue, R., Fernando. L., Bayardo, G. y Mauricio H. (2014). ¿Para qué ciencia, tecnología, y sociedad en las universidades? Guayaquil: Universidad Espirito Santo, Universidad Católica de Guayaquil, Universidad Ecotec, Universidad de La Habana.

Núñez, J. (1999): La ciencia y la Tecnología como Procesos Sociales. Lo que la Educación Científica no debería olvidar. Editorial Félix Varela, La Habana. 
Osorio, C. (2002) La educación científica y tecnológica desde el enfoque en Ciencia, Tecnología y Sociedad: aproximaciones y experiencias para la educación secundaria. Revista Ibero-Americana de Educação, 28, (6), pp.1-81

Osorio, C. y Martins, I.(2011) La Educación Científica y Tecnológica para el Espacio Iberoamericano de Conocimiento. Ciencia, Tecnología y Universidad en Iberoamérica. Buenos Aires, pp. 119-141.

Pedrosa, M. y Martins, I. (2002). Integración de CTS en el sistema educativo portugués. En P. Membiela (Ed.): Enseñanza de las ciencias desde la perspectiva ciencia-tecnología-sociedad. Formación científica para la ciudadanía. Madrid; Editorial Narcea.

Pinheiro, N. y Bazzo, W. (2005); Educação crítico-reflexiva para um Ensino Médio científico tecnológico: a contribuição do enfoque CTS para o ensino- aprendizagem do conhecimento matemático. Florianópolis, 2005. (Tese de Doutorado em Educação: Ensino de Ciências sem publicar) - Universidade Federal de Santa Catarina, Brasil.

Pinheiro, N., Silveira, R. y Bazzo, W. (2007) Ciência, Tecnologia e Sociedade: a relevância do enfoque CTS para o contexto do Ensino Médio. Revista Ciência e educação, Bauru, 13, (1), pp.71-84. Disponível em:http:// www.scielo.br/pdf/ ciedu/v13n1/v13n1a05.pdf. Acesso em: 18 abr. 2011.

Santos, W. (2008) Educação Científica Humanística em uma perspectiva Freireana: resgatando a função do ensino de CTS. Revista Alexandria 1, (1), pp.109-131.

Villegas, M. y González, F. (2011). La investigación cualitativa de la vida cotidiana. Medio para la construcción de conocimiento sobre lo social a partir de lo individual. Revista Psicoperspectivas. Individuo y Sociedad, 10( 2), pp. 35-59.

Wongo, G., Faustino, A. y Pérez E. (2013). El contexto angolano de formación matemática: Un problema que se arrastra desde la base. Avaliação: Revista da Educação Superior, 18(2), 487-499. DOI: http:// dx.doi.org/10.1590/S1414-40772013000200013

\section{Notas}

[1] Epistemología. Ciencia que estudia los procesos cognitivos humanos.

\section{BY-NC-ND}

